\title{
Archimedean-Compensatory Fuzzy Logic Systems
}

\author{
Rafael A. Espin-Andrade \\ Engineering and Technology University, Universidad de Occidente, \\ Blvd Lola Beltrán S/N, Culiacán, Sinaloa, CP 80020, Mexico \\ E-mail: rafaelalejandroespinandrade@gmail.com;www.udo.mx
}

Erick González Caballero

Mathematical Center for Technical Sciences, Higher Technical University of Havana, 27 Calle 114 \# 11901 e/ Ciclovía y Rotonda, Marianao,

Havana, Cuba

E-mail: erickgc@yandex.com; www.cujae.edu.cu

Witold Pedrycz

Department of Electrical \& Computer Engineering, University of Alberta

Edmonton T6R 2V4 AB, Canada

Faculty of Engineering, King Abdulaziz University

Jeddah, 21589, Saudi Arabia

and

Systems Research Institute, Polish Academy of Sciences

Warsaw, Poland

E-mail: wpedrycz@ualberta.ca;www.ualberta.ca

Eduardo R. Fernández González

Autonomous University of Sinaloa, Calzada de las Américas y Boulevard Universitarios $s / n$

Culiacán Rosales, Sinaloa 80040, Mexico

E-mail:eddyf@uas.uasnet.mx; www.uas.edu.mx

Received 5 August 2015

Accepted 30 October 2015

\begin{abstract}
The paper aims to define a new kind of logic, referred to as Archimedean-Compensatory Logic, which is constructed from the unification of two different fuzzy logic systems, namely a continuous Archimedean fuzzy logic and a compensatory fuzzy logic. The paper introduces basic definitions and properties of this new theory. Continuous Archimedean logic is a t-norm and t-conorm logic system and Compensatory Fuzzy Logic can be obtained from quasi-arithmetic mean operators. We will prove the property that the preference over a pair of truth-value vectors is the same for certain predicates in the Compensatory Fuzzy Logic and the Continuous Archimedean Logic.
\end{abstract}

Keywords: Archimedean fuzzy logic, Compensatory fuzzy logic. 


\section{Introduction}

Two ways of defining logic systems are t-norm and tconorm approach and Compensatory Fuzzy Logic (CFL). T-norm and t-conorm have been widely studied and applied by fuzzy logic community; see Ref. 1, even though sometimes this notion does not fully correspond to the way in which we make decisions.

On the other hand, CFL, Ref. 2, satisfies the idea of allowing compensation under certain rules, which is also an interesting approach to model decision-making realized by humans.

This paper aims to show that both approaches can be theoretically compatible. That is to say, we will show that the quartet formed by one universal and one existential predicate based on a continuous Archimedean t-norm and others two, one universal and one existential predicates based on a compensatory system, increases the power for modeling decision making. We will be referring to such logic systems Archimedean-Compensatory logics.

In Refs. 3-4, for example, we can find an earlier approach to define a compensatory AND by a t-norm conjunction and a t-conorm disjunction. Nevertheless, the author doesn't use the notion of relationship between two logic systems, one compensatory and the other one, Archimedean.

Also, Refs. 5-8 contain approaches for defining compensatory operators from t-norm and t-conorm operators, by means of iteration in Refs.7-8, but the limitations are the same which we explained above.

There is a mathematical relationship between the way of generating a continuous Archimedean t-norm and such for generating its corresponding compensatory operator, which is the justification of the ideas expressed above.

Also, we introduce the concept of compatibility of two fuzzy logic systems and we prove that for each continuous Archimedean logic based on one t-norm and t-conorm, and its compatible CFL, there exists the same order for the truth value of the predicates.

The Archimedean universal quantifier and its corresponding existential quantifier represent the tendency to no refute a decision making represented by a predicate, respectively. Equivalently for the CFL, the pair formed by a CFL universal quantifier and a CFL existential quantifier represents the affirmation.

The aim of this paper is to reveal the coherence that exists between the t-norm and t-conorm theory and the
CFL theory. Some examples will help illustrate the usefulness of such unification in some applications.

\section{Underlying definitions}

A t-norm is a function $\mathrm{T}:[0,1]^{2} \rightarrow[0,1]$, which satisfies the following axioms, see Ref. 1:

- $\mathrm{T}(\mathrm{a}, \mathrm{b})=\mathrm{T}(\mathrm{b}, \mathrm{a})(\mathrm{T} 1)$ Commutativity,

- $\mathrm{T}(\mathrm{a}, \mathrm{b}) \leq \mathrm{T}(\mathrm{c}, \mathrm{d})$ if $\mathrm{a} \leq \mathrm{c}$ and $\mathrm{b} \leq \mathrm{d}$ (T2) Monotonicity,

- $\quad \mathrm{T}(\mathrm{a}, \mathrm{T}(\mathrm{b}, \mathrm{c}))=\mathrm{T}(\mathrm{T}(\mathrm{a}, \mathrm{b}), \mathrm{c})(\mathrm{T} 3)$ Associativity

- $\mathrm{T}(\mathrm{a}, 1)=\mathrm{a}(\mathrm{T} 4)$ One as identity.

A t-conorm is a function $\mathrm{S}:[0,1]^{2} \rightarrow[0,1]$, which satisfies the following axioms:

- $\quad \mathrm{S}(\mathrm{a}, \mathrm{b})=\mathrm{S}(\mathrm{b}, \mathrm{a})(\mathrm{S} 1)$ Commutativity,

- $\mathrm{S}(\mathrm{a}, \mathrm{b}) \leq \mathrm{S}(\mathrm{c}, \mathrm{d})$ if $\mathrm{a} \leq \mathrm{c}$ and $\mathrm{b} \leq \mathrm{d}(\mathrm{S} 2)$ Monotonicity,

- $\quad S(a, S(b, c))=S(S(a, b), c)(S 3)$ Associativity,

- $\mathrm{T}(\mathrm{a}, 0)=\mathrm{a}(\mathrm{S} 4)$ Zero as identity.

There is a classical property which matches t-norm and t-conorm; it is the De Morgan's Laws.

$\neg \mathrm{T}(\mathrm{a}, \mathrm{b})=\mathrm{S}(\neg \mathrm{a}, \neg \mathrm{b})$ and $\neg \mathrm{S}(\mathrm{a}, \mathrm{b})=\mathrm{T}(\neg \mathrm{a}, \neg \mathrm{b})$.

The most usual negation operator is $\neg \mathrm{a}=1-\mathrm{a}$

A t-norm $\mathrm{T}:[0,1]^{2} \rightarrow[0,1]$ is a continuous t-norm if and only if for every pair of convergent sequences $\left\{x_{n}\right\}_{n=1}^{\infty}$ and $\left\{y_{n}\right\}_{n=1}^{\infty}$ in $[0,1], \lim _{n \rightarrow \infty} T\left(x_{n}, y_{n}\right)=$ $T\left(\lim _{n \rightarrow \infty} x_{n}, \lim _{n \rightarrow \infty} y_{n}\right)$.

A $\mathrm{t}$-norm $\mathrm{T}^{\mathrm{n}}:[0,1]^{2} \rightarrow[0,1]$ is said to be Archimedean if and only if it satisfies one of the following conditions:

1. For every $x \in(0,1), T(x, x)<x$

2. For every $x, y \in(0,1)$ there is a natural number $n$ such that

$\mathrm{T}(\mathrm{x}, \mathrm{x}, \ldots, \mathrm{x})<\mathrm{y}$ $n$ times

3. For every $x \in(0,1)$ there is a natural number $n$ such that $\mathrm{T}(\mathrm{x}, \mathrm{x}, \ldots, \mathrm{x})=0$

$n$ times

Here we prefer definition number 1.

\section{Properties}

1. Every t-norm $\mathrm{T}:[0,1]^{2} \rightarrow[0,1]$, satisfies $\mathrm{T}(\mathrm{x}, \mathrm{y}) \leq$ $\min \{x, y\}$

2. Every t-conorm $S:[0,1]^{2} \rightarrow[0,1]$, satisfies $\mathrm{S}(\mathrm{x}, \mathrm{y}) \geq \max \{\mathrm{x}, \mathrm{y}\}$

3. $\min (x, y)$ is the unique continuous idempotent $t-$ norm and the maximum function of the family of $t-$ norm operators.

4. $\max (\mathrm{x}, \mathrm{y})$ is the unique continuous idempotent $\mathrm{t}$ conorm and the minimum function of the family of t-conorm operators. 
5. One consequence of the precedent properties is that every t-norm (t-conorm) belongs to only one of two subsets, the singleton of the min t-norm (the max tconorm) or the set of non-idempotent t-norms ( $t$ conorms).

6. For every continuous Archimedean t-norm there exists a non-increasing continuous function $f:[0,1] \rightarrow[0,+\infty]$ satisfying $f(1)=0$, such that:

$$
\mathrm{T}\left(x_{1}, \cdots, x_{n}\right)=\mathrm{f}^{(-1)}\left(\sum_{\mathrm{i}=1}^{\mathrm{n}} \mathrm{f}\left(x_{i}\right)\right)
$$

Where,

$$
\mathrm{f}^{(-1)}(\mathrm{z})=\left\{\begin{array}{c}
\mathrm{f}^{-1}(\mathrm{z}), \text { if } \mathrm{z} \in[0, \mathrm{f}(0)] \\
0, \text { if }(\mathrm{f}(0),+\infty]
\end{array}\right.
$$

7. An equivalent property exists for t-conorms.

Let $n$ be a negation operator from $[0,1]$ to $[0,1]$, or a strictly decreasing operator fulfilling $n(n(\mathrm{x})=\mathrm{x}, n(0)=1$ and $n(1)=0$.

\section{Definition 1}

An Archimedean Logic is a trio (c, $d, n)$ of operators, where $c$ is a continuous Archimedean t-norm, $d$ is its corresponding continuous Archimedean t-conorm and $n$ is the negation operator.

From now let $\mathbf{x}=\left(x_{1}, x_{2}, \ldots, x_{\mathrm{n}}\right), \quad \mathbf{y}=\left(\mathrm{y}_{1}, \mathrm{y}_{2}, \ldots, \mathrm{y}_{\mathrm{n}}\right)$, $\mathbf{z}=\left(\mathbf{z}_{1}, \mathbf{z}_{2}, \ldots, \mathbf{z}_{\mathrm{n}}\right)$ be any element of the Cartesian product $[0,1]^{\mathrm{n}}$.

A 4-tuple of continuous operators $(c, d, o, n), c$ and $d$ from $[0,1]^{\mathrm{n}}$ into $[0,1]$, the operator $o$ from $[0,1]^{2}$ to $[0,1]$ and $n$ a negation operator, constitute a Compensatory Fuzzy Logic (CFL), see Ref. 2, if the following group of axioms is satisfied:

(i)

$$
\begin{aligned}
& \text { Compensation Axiom: } \\
& \min \left\{x_{1}, x_{2}, \cdots, x_{n}\right\} \leq c\left(x_{1}, x_{2}, \cdots, x_{n}\right) \\
& \leq \max \left\{x_{1}, x_{2}, \cdots, x_{n}\right\}
\end{aligned}
$$$$
\text { Commutativity or Symmetry Axiom: }
$$$$
c\left(x_{1}, x_{2}, \ldots, x_{\mathrm{i}}, \ldots, x_{\mathrm{j}}, \ldots, x_{\mathrm{n}}\right)=
$$$$
c\left(x_{1}, x_{2}, \ldots, x_{\mathrm{j}}, \ldots, x_{\mathrm{i}}, \ldots, x_{\mathrm{n}}\right) \text {. }
$$

Strict Growth Axiom: If $x_{1}=\mathrm{y}_{1}, x_{2}=\mathrm{y}_{2}, \ldots$,

$x_{\mathrm{i}-1}=\mathrm{y}_{\mathrm{i}-1}, \quad x_{\mathrm{i}+1}=\mathrm{y}_{\mathrm{i}+1}, \ldots, x_{\mathrm{n}}=\mathrm{y}_{\mathrm{n}}$ are unequal to zero, and $x_{\mathrm{i}}>\mathrm{y}_{\mathrm{i}}$ then $c\left(x_{1}, x_{2}, \ldots, x_{\mathrm{n}}\right)>c\left(\mathrm{y}_{1}, \mathrm{y}_{2}, \ldots, \mathrm{y}_{\mathrm{n}}\right)$
Veto Axiom: If $x_{\mathrm{i}}=0$ for an i then $c(\boldsymbol{x})=0$.

Fuzzy Reciprocity Axiom: $o(x, \mathrm{y})=n[o(\mathrm{y}, x)]$

Fuzzy Transitivity Axiom: If $o(x, y) \geq 0.5$ and $o(\mathrm{y}, \mathrm{z}) \geq 0.5, \quad$ then $o(x, \mathrm{z})$ $\geq \max (o(x, \mathrm{y}), o(\mathrm{y}, \mathrm{z}))$

De Morgan's Laws:

$$
\begin{aligned}
& n\left(c\left(x_{1}, x_{2}, \cdots, x_{n}\right)\right) \\
& =d\left(n\left(x_{1}\right), n\left(x_{2}\right), \cdots, n\left(x_{n}\right)\right)
\end{aligned}
$$

$$
\begin{aligned}
& n\left(d\left(x_{1}, x_{2}, \cdots, x_{n}\right)\right) \\
& =c\left(n\left(x_{1}\right), n\left(x_{2}\right), \cdots, n\left(x_{n}\right)\right)
\end{aligned}
$$

$d$ operator satisfies the properties below:

1. Compensation Property:

2. Symmetry Property:

$$
\begin{gathered}
\min \left\{x_{1}, x_{2}, \cdots, x_{n}\right\} \leq d\left(x_{1}, x_{2}, \cdots, x_{n}\right) \\
\leq \max \left\{x_{1}, x_{2}, \cdots, x_{n}\right\}
\end{gathered}
$$

$d\left(x_{1}, x_{2}, \ldots, x_{\mathrm{i}}, \ldots, x_{\mathrm{j}}, \ldots, x_{\mathrm{n}}\right)=d\left(x_{1}, x_{2}, \ldots, x_{\mathrm{j}}, \ldots, x_{\mathrm{i}}, \ldots, x_{\mathrm{n}}\right)$.

3. Strict Growth Property:

If $x_{1}=\mathrm{y}_{1}, x_{2}=\mathrm{y}_{2}, \ldots$,

$$
\begin{aligned}
& x_{\mathrm{i}-1}=\mathrm{y}_{\mathrm{i}-1}, x_{\mathrm{i}+1}=\mathrm{y}_{\mathrm{i}+1}, \ldots, x_{\mathrm{n}}=\mathrm{y}_{\mathrm{n}} \text { are unequal to } \\
& \text { one, } \quad \text { and } x_{\mathrm{i}}>\mathrm{y}_{\mathrm{i}} \\
& d\left(x_{1}, x_{2}, \ldots, x_{\mathrm{n}}\right)>d\left(\mathrm{y}_{1}, \mathrm{y}_{2}, \ldots, \mathrm{y}_{\mathrm{n}}\right)
\end{aligned}
$$

4. Veto Axiom: If $x_{\mathrm{i}}=1$ for an $\mathrm{i}$ then $d(\mathbf{x})=1$

5. $\quad c(\mathrm{a}, \mathrm{a}, \ldots, \mathrm{a})=\mathrm{a}, d(\mathrm{a}, \mathrm{a}, \ldots, \mathrm{a})=\mathrm{a}$, idempotency of $c$ and $d$ operators.

Operators $c$ and $d$ are called conjunction and disjunction, respectively. The operator $o$ is called fuzzy strict order.

A family of CFL is the quasi-arithmetic means, which includes for example the geometric mean. They are operators of the form:

$$
\begin{aligned}
& M_{f}\left(x_{1}, x_{2}, \cdots, x_{n}\right) \\
= & f^{-1}\left(\frac{\sum_{i=1}^{n} f\left(x_{i}\right)}{n}\right)(3)
\end{aligned}
$$

$\mathrm{f}$ is a strictly monotone continuous function, which is extended to the non-defined points by using the corresponding limit.

These operators satisfy Axioms i-iii. In addition, if we have that for all $\mathrm{i} \in\{1,2, \ldots, \mathrm{n}\}$, $\lim _{x_{i} \rightarrow 0} M_{f}\left(x_{1}, x_{2}, \cdots, x_{n}\right)=0$, we obtain axiom iv as well. Then, taking Eqs. (4), (5) and (6)

$$
\begin{gathered}
d\left(x_{1}, x_{2}, \cdots, x_{n}\right)= \\
1-f^{-1}\left(\frac{\sum_{i=1}^{n} f\left(1-x_{i}\right)}{n}\right) \\
n(\mathbf{x})=1-\mathbf{x} \quad(4) \\
o(\mathbf{x}, \mathbf{y})=0.5[c(\mathbf{x})-c(\mathbf{y})]+0.5 \quad(6),
\end{gathered}
$$

we have a class of Compensatory Logics referred to as Quasi Arithmetic Mean Based Compensatory Logic (QAMBCL).

A special case of Eq. (3) is obtained for $f(x)=x^{p}$, where $\mathrm{p} \in(-\infty, 0]$, see definitions presented in Eqs. (7) and (8).

$$
\begin{gathered}
c\left(x_{1}, x_{2}, \cdots, x_{n}\right)=\left(\frac{\sum_{i=1}^{n} x_{i}^{p}}{n}\right)^{1 / p} \\
d\left(x_{1}, x_{2}, \cdots, x_{n}\right)=1-\left(\frac{\sum_{i=1}^{n}\left(1-x_{i}\right)^{p}}{n}\right)^{1 / p}
\end{gathered}
$$

Let us note that Eqs. (7) and (8) tend to the geometric mean and the dual of the geometric mean, respectively, in case that $p=0$. See, Eqs. (9) and (10).

$$
\begin{gathered}
c\left(x_{1}, x_{2}, \cdots, x_{n}\right)=\sqrt[n]{\prod_{i=1}^{n} x_{i}} \\
d\left(x_{1}, x_{2}, \cdots, x_{n}\right)=1-\sqrt[n]{\prod_{i=1}^{n}\left(1-x_{i}\right)}
\end{gathered}
$$


This last compensatory logic with the operators of conjunction and disjunction defined like in Eqs. (9) and (10), is called Geometric Mean based Compensatory Logic (GMBCL).

Let us show that GMBCL satisfies the axioms of CFL. Evidently, (9) fulfills axiom (ii). If we change the order of the elements of vector $\left(x_{1}, x_{2}, \ldots, x_{\mathrm{n}}\right)$, the geometric mean value will remain equally.

If the truth value of one element is 0 , then the geometric mean is 0 , therefore (9) satisfies the veto axiom, corresponding to one classic axiom of the bivalent logic for conjunction.

An equivalent proof is valid for the veto axiom for the disjunction in (10).

Because of the continuity of the geometric mean and also the non-decreasing monotony of this function, if we increase the truth value of one element, then the value of the geometric mean increases. Hence, (9) satisfies the axiom (iii) and (10) satisfies the property 3. These two axioms are important to decision making, where the capacity for ordering is essential.

$c(a, a, \cdots, a)=\sqrt[n]{\prod_{i=1}^{n} a}=a$ and $d(a, a, \cdots, a)=1-$ $\sqrt[n]{\prod_{i=1}^{n}(1-a)}=1-(1-a)=a$. Therefore, (9) and (10) satisfy idempotency, for more details see Ref. 2.

Idempotency is necessary if we think the propositional formulas like utility functions of Decision Theory and then, this axiom corresponds to a cardinal measure. From this point of view, t-norms and t-conorms operators offer just ordinal measure operators.

Applying $\mathrm{n}$ times the Strict Growth Axiom we can prove the compensation in (9) and (10).

We defined the negation operator in the most usual way. The De Morgan Laws are classical and easy to prove.

The order operator is included in CFL due to the importance for ordering in Decision Making.

Let us note that the family of CFL is bigger than GMBCL and bigger than QAMBCL family. These two families are special cases of (3).

\section{Archimedean-Compensatory Logic}

This section explains the theory of the ArchimedeanCompensatory Logic, as well as the definitions and the theoretical demonstrations of its properties

\section{Definition 2}

Two fuzzy logic systems, $L_{1}$ and $L_{2}$, it is said they are compatible for the formula $p$ of the propositional calculus if and only if for every pair of vectors $\boldsymbol{x}=$ $\left(x_{1}, x_{2}, \ldots, x_{n}\right)$ and $\boldsymbol{y}=\left(y_{1}, y_{2}, \ldots, y_{n}\right)$ into $[0,1]^{n}$ they satisfy the property: $p_{L 1}(\boldsymbol{x})<p_{L 1}(\boldsymbol{y})$ if and only ifp $p_{L 2}(\boldsymbol{x})<p_{L 2}(\boldsymbol{y})$, where $p_{L 1}$ and $p_{L 2}$, represent the calculus for the formula p by using logic systems $L_{1}$ and $L_{2}$, respectively.

\section{Definition 3}

Two fuzzy logic systems, $L_{1}$ and $L_{2}$ it is said they are compatible if and only if they are compatible for every formula of the propositional calculus.

\section{Proposition 1}

Let be $f(x)$ a strictly monotone real-valued function which generates a continuous Archimedean t-norm, then, the t-norm and its corresponding quasi-arithmetic conjunction operator (the one defined by the same function f) satisfy the property of compatibility of definition 3 for the conjunction and disjunction operators.

Proof. First, we will prove it for the conjunction operator.

Let be $\mathrm{f}(\mathrm{x})$ an strictly decreasing function and $\mathrm{c}_{\mathrm{T}}(\mathbf{x})=$ $\mathrm{f}^{(-1)}\left(\sum_{\mathrm{i}=1}^{\mathrm{n}} \mathrm{f}\left(\mathrm{x}_{\mathrm{i}}\right)\right)>\mathrm{c}_{\mathrm{T}}(\mathrm{y})=\mathrm{f}^{(-1)}\left(\sum_{\mathrm{i}=1}^{\mathrm{n}} \mathrm{f}\left(\mathrm{y}_{\mathrm{i}}\right)\right)$

Evaluating $\mathrm{f}(\mathrm{x})$ in the inequality above, $\sum_{\mathrm{i}=1}^{\mathrm{n}} \mathrm{f}\left(\mathrm{x}_{\mathrm{i}}\right)<$ $\sum_{\mathrm{i}=1}^{\mathrm{n}} \mathrm{f}\left(\mathrm{y}_{\mathrm{i}}\right)$.

Therefore, $\quad \frac{\sum_{\mathrm{i}=1}^{\mathrm{n}} \mathrm{f}\left(\mathrm{x}_{\mathrm{i}}\right)}{\mathrm{n}}<\frac{\sum_{\mathrm{i}=1}^{\mathrm{n}} \mathrm{f}\left(\mathrm{y}_{\mathrm{i}}\right)}{\mathrm{n}}$ and $\quad \mathrm{c}_{\mathrm{C}}(\mathrm{x})=$ $\mathrm{f}^{(-1)}\left(\frac{\sum_{\mathrm{i}=1}^{\mathrm{n}} \mathrm{f}\left(\mathrm{x}_{\mathrm{i}}\right)}{\mathrm{n}}\right)>\mathrm{c}_{\mathrm{C}}(\mathrm{y})=\mathrm{f}^{(-1)}\left(\frac{\sum_{\mathrm{i}=1}^{\mathrm{n}} \mathrm{f}\left(\mathrm{y}_{\mathrm{i}}\right)}{\mathrm{n}}\right), \quad$ because $\mathrm{f}(\mathrm{x})$ is a decreasing function if and only if $\mathrm{f}^{(-1)}(\mathrm{y})$ is decreasing too.

Let be $\mathrm{f}(\mathrm{x})$ an strictly increasing function and $\mathrm{c}_{\mathrm{T}}(\mathrm{x})=$ $\mathrm{f}^{(-1)}\left(\sum_{\mathrm{i}=1}^{\mathrm{n}} \mathrm{f}\left(\mathrm{x}_{\mathrm{i}}\right)\right)>\mathrm{c}_{\mathrm{T}}(\mathrm{y})=\mathrm{f}^{(-1)}\left(\sum_{\mathrm{i}=1}^{\mathrm{n}} \mathrm{f}\left(\mathrm{y}_{\mathrm{i}}\right)\right)$,

evaluating $f(x)$ in the inequality above, $\sum_{i=1}^{n} f\left(x_{i}\right)>$ $\sum_{i=1}^{n} f\left(y_{i}\right)$.

Therefore, $\quad \frac{\sum_{\mathrm{i}=1}^{\mathrm{n}} \mathrm{f}\left(\mathrm{x}_{\mathrm{i}}\right)}{\mathrm{n}}>\frac{\sum_{\mathrm{i}=1}^{\mathrm{n}} \mathrm{f}\left(\mathrm{y}_{\mathrm{i}}\right)}{\mathrm{n}}$ and $\quad \mathrm{c}_{\mathrm{C}}(\mathrm{x})=$ $\mathrm{f}^{(-1)}\left(\frac{\sum_{\mathrm{i}=1}^{\mathrm{n}} \mathrm{f}\left(\mathrm{x}_{\mathrm{i}}\right)}{\mathrm{n}}\right)>\mathrm{c}_{\mathrm{C}}(\mathrm{y})=\mathrm{f}^{(-1)}\left(\frac{\sum_{\mathrm{i}=1}^{\mathrm{n}} \mathrm{f}\left(\mathrm{y}_{\mathrm{i}}\right)}{\mathrm{n}}\right), \quad$ because $\mathrm{f}(\mathrm{x})$ is an increasing function if and only if $\mathrm{f}^{(-1)}(\mathrm{y})$ is increasing too.

The proof of the other implication, that is to say, if $\mathrm{c}_{\mathrm{C}}(\mathrm{x})>\mathrm{c}_{\mathrm{C}}(\mathrm{y})$, then $\mathrm{c}_{\mathrm{T}}(\mathrm{x})>\mathrm{c}_{\mathrm{T}}(\mathrm{y})$, is similar.

The proof for the disjunction is similar if $\mathrm{f}$ is substituted by the corresponding function for the disjunction operator.

\section{Proposition 2}

Let be $f(x)$ a strictly monotone real-valued function which generates a continuous Archimedean t-norm, then, the t-norm and its corresponding quasi-arithmetic conjunction operator satisfy the property of compatibility of definition 3 for the implication and equivalence operators, if they are defined like $i(x, y)=d(n(x), y)$ and $e(x, y)=c(i(x, y), i(y, x))$, respectively and where the negation is $n(x)=1-x$.

Proof. First, let us prove it for the implication.

Let us note that, according to the definitions above, there is a non-decreasing (non-increasing) function $\mathrm{g}$ 
corresponding to a Continuous Archimedean Logic, such that $i_{T}(\mathrm{x}, \mathrm{y})=\mathrm{g}^{(-1)}(\mathrm{g}(1-\mathrm{x})+\mathrm{g}(\mathrm{y}))$.

Suppose that $i_{T}\left(\mathrm{x}_{1}, \mathrm{y}_{1}\right)<i_{T}\left(\mathrm{x}_{2}, \mathrm{y}_{2}\right)$ (index $\mathrm{T}$ means that the implication is calculated with a Continuous Archimedean t-conorm), and hence, $\mathrm{g}^{(-1)}\left(\mathrm{g}\left(1-\mathrm{x}_{1}\right)+\right.$ $\left.g\left(y_{1}\right)\right)<g^{(-1)}\left(g\left(1-x_{2}\right)+g\left(y_{2}\right)\right)$.

If $g$ is a non-increasing function then, $g\left(1-x_{1}\right)+$ $\mathrm{g}\left(\mathrm{y}_{1}\right)>\mathrm{g}\left(1-\mathrm{x}_{2}\right)+\mathrm{g}\left(\mathrm{y}_{2}\right)$ and $\quad \frac{\mathrm{g}\left(1-\mathrm{x}_{1}\right)+\mathrm{g}\left(\mathrm{y}_{1}\right)}{2}>$ $\frac{\mathrm{g}\left(1-\mathrm{x}_{2}\right)+\mathrm{g}\left(\mathrm{y}_{2}\right)}{2}$, therefore $\mathrm{g}^{(-1)}\left(\frac{\mathrm{g}\left(1-\mathrm{x}_{1}\right)+\mathrm{g}\left(\mathrm{y}_{1}\right)}{2}\right)<$ $\mathrm{g}^{(-1)}\left(\frac{\mathrm{g}\left(1-\mathrm{x}_{2}\right)+\mathrm{g}\left(\mathrm{y}_{2}\right)}{2}\right)$, which means that $i_{c}\left(\mathrm{x}_{1}, \mathrm{y}_{1}\right)$ $<i_{c}\left(\mathrm{x}_{2}, \mathrm{y}_{2}\right)$ for the respective compensatory implication. If $\mathrm{g}$ is a non-decreasing function then, $\mathrm{g}\left(1-\mathrm{x}_{1}\right)+$ $\mathrm{g}\left(\mathrm{y}_{1}\right)<\mathrm{g}\left(1-\mathrm{x}_{2}\right)+\mathrm{g}\left(\mathrm{y}_{2}\right)$ and $\frac{\mathrm{g}\left(1-\mathrm{x}_{1}\right)+\mathrm{g}\left(\mathrm{y}_{1}\right)}{2}<$ $\frac{\mathrm{g}\left(1-\mathrm{x}_{2}\right)+\mathrm{g}\left(\mathrm{y}_{2}\right)}{2}, \quad$ therefore $\quad \mathrm{g}^{(-1)}\left(\frac{\mathrm{g}\left(1-\mathrm{x}_{1}\right)+\mathrm{g}\left(\mathrm{y}_{1}\right)}{2}\right)<$ $\mathrm{g}^{(-1)}\left(\frac{\mathrm{g}\left(1-\mathrm{x}_{2}\right)+\mathrm{g}\left(\mathrm{y}_{2}\right)}{2}\right)$, which means that $i_{c}\left(\mathrm{x}_{1}, \mathrm{y}_{1}\right)$ $<i_{c}\left(\mathrm{x}_{2}, \mathrm{y}_{2}\right)$.

Similarly it is demonstrated that $i_{c}\left(\mathrm{x}_{1}, \mathrm{y}_{1}\right)<i_{c}\left(\mathrm{x}_{2}, \mathrm{y}_{2}\right)$ implies $i_{T}\left(\mathrm{x}_{1}, \mathrm{y}_{1}\right)<i_{T}\left(\mathrm{x}_{2}, \mathrm{y}_{2}\right)$.

The proof for the equivalence operator $e$ results from the proof for the implication operator and proposition 1 for the conjunction operator.

\section{Proposition 3}

Every continuous Archimedean logic and its corresponding Compensatory Fuzzy Logic are compatible for every propositional formula formed recursively by atoms formulas, conjunction, disjunction, negation, natural implication and equivalence operators.

Proof. If $\mathrm{p}$ is formed by $\mathrm{n}$ operators belonging to the set of operators established in the hypothesis, then, applying recursively proposition 1 or proposition 2 from the most external operator in the formula to the most internal, then it can be proved this proposition.

\section{Remark 1}

The set of operators stated in proposition 3 can be extended by including the strict order operator of formula (6). This inclusion doesn't affect the result of proposition 3. Let us note that this operator doesn't depend on the logic system.

\section{Definition 4}

An Archimedean-Compensatory Logic is the sextet $\left(c_{T}, c_{C}, d_{T}, d_{C}, O, n\right)$ of operators such that $\left(c_{T}, d_{T}, n\right)$ is an Archimedean Logic according to definition 1, and $\left(c_{C}, d_{C}, o, n\right)$ is a Compensatory Fuzzy Logic, such that,the Archimedean logic and the Compensatory Logic are compatible, according to definition 3.

\section{Remark 2}

The condition of compatibility in definition 3 is well defined because it is a consequence of propositions 1 and 2 .

\section{Remark 3}

According to proposition 1 applied to definition $4, c_{T}$ and $c_{C}$ maintain the same order. Therefore, the Archimedean-Compensatory Logic satisfies that this is compatible and indifferent for the order to calculate with the t-norm conjunction or to the compensatory conjunction operator.

\section{Example 1}

One Archimedean-Compensatory logic is obtained from the probabilistic logic, see Ref. 1, and the GMBCL, see Ref. 2, the definition of the operators is summarized in the following table:

Table 1. Operators of the probabilistic logic system and the GMBCL

\begin{tabular}{lcc}
\hline Operator & Probabilistic logic system & GMBCL system \\
\hline Conjunction & $c\left(\mathrm{x}_{1}, \mathrm{x}_{2}\right)=\mathrm{x}_{1} \cdot \mathrm{x}_{2}$ & $c(\mathrm{x})=\sqrt{\mathrm{n}_{\mathrm{i}=1}^{\mathrm{n}} \mathrm{x}_{\mathrm{i}}}$ \\
Disjunction & $d\left(\mathrm{x}_{1}, \mathrm{x}_{2}\right)=\mathrm{x}_{1}+\mathrm{x}_{2}$ & $d(\mathrm{x})=$ \\
(dual of the & $-\mathrm{x}_{1} \cdot \mathrm{x}_{2}$ & $\left.1-\sqrt[\mathrm{n}]{\pi_{\mathrm{i}=1}^{\mathrm{n}}\left(1-\mathrm{x}_{\mathrm{i}}\right.}\right)$ \\
conjunction) & $1-\mathrm{x}$ & $1-\mathrm{x}$ \\
Negation & Reichenbach implication & Natural \\
Implication & $i\left(\mathrm{x}_{1}, \mathrm{x}_{2}\right)=1-\mathrm{x}_{1}+\mathrm{x}_{1} \mathrm{x}_{2}$ & implication \\
$\left(d\left(n\left(x_{1}\right), x_{2}\right)\right)$ & & $i\left(\mathrm{x}_{1}, \mathrm{x}_{2}\right)=$ \\
& & $1-\sqrt{\mathrm{x}_{1}-\mathrm{x}_{1} \mathrm{x}_{2}}$ \\
& & $\sqrt{i\left(\mathrm{x}_{1}, \mathrm{x}_{2}\right) \cdot i\left(\mathrm{x}_{2}, \mathrm{x}_{1}\right.}$ \\
Equivalence & & \\
$c\left(i\left(x_{1}, X_{2}\right)\right.$, & & \\
$\left.i\left(x_{2}, x_{1}\right)\right)$ & &
\end{tabular}

Conjunction and disjunction operators corresponding to both logic systems can be generated by the same additive generators, $\mathrm{f}(\mathrm{u})=-\ln (\mathrm{u})$ for conjunction and $\mathrm{f}(\mathrm{u})=-\ln (1-\mathrm{u})$ for disjunction, $\mathrm{f}^{(-1)}(\mathrm{u})=\mathrm{e}^{-\mathrm{u}}$.

Thus, this example of Archimedean-Compensatory Logic is generated by the pair of functions $-\ln (u)$ and $\ln (1-u)$. They satisfy proposition 3 and it is compatible to calculate with probabilistic logic or GMBCL, in the sense to maintain the order of the predicates, which are formed by the operators of the Table 1 .

\section{Example 2}

Table 2 is a summary about some indexes of the Mexican Economy, see Ref. 11. 
Table 2. Behavior of some index of the Mexican Economy for seven years

\begin{tabular}{|c|c|c|c|c|}
\hline & $\mathrm{A}_{1}$ (Inflation) & $\mathrm{A}_{2}(\mathrm{GDP})$ & $\begin{array}{l}\mathrm{A}_{3} \text { (Tax of } \\
\text { exchange } \\
\text { Peso/Dollar) }\end{array}$ & $\begin{array}{l}\mathrm{A}_{4} \\
\text { (Demand) }\end{array}$ \\
\hline \multicolumn{5}{|c|}{ Year/Attribute } \\
\hline 1 & 11.01 & 2 & 2.3 & 32350 \\
\hline 2 & 7.06 & 4.5 & 4.8 & 31305 \\
\hline 3 & 52 & -6.2 & 5.6 & 28083 \\
\hline 4 & 27.7 & 5.2 & 6.5 & 33408 \\
\hline 5 & 15.7 & 7 & 6.95 & 44987 \\
\hline 6 & 18.8 & 4.1 & 10.05 & 54344 \\
\hline 7 & 17.2 & 2.9 & 11.4 & 56830 \\
\hline
\end{tabular}

The truth values calculated for the indexes of the Mexican Economy in Table 2 are summarized in Table 3.

Table 3. Truth values of the elements of the Mexican Economy in Table 2

\begin{tabular}{lllll}
\hline Year/ & $\mathrm{A}_{1}$ (Inflation) & $\mathrm{A}_{2}$ (GDP) & $\begin{array}{l}\mathrm{A}_{3} \text { (Tax of } \\
\text { exchange } \\
\text { Pttribute }\end{array}$ & $\begin{array}{l}\mathrm{A}_{4} \\
\text { (Demand) }\end{array}$ \\
\hline 1 & 0.01 & 0.5 & 0.987 & 0.557 \\
2 & 0.17 & 0.99 & 0.883 & 0.388 \\
3 & $2.33 \mathrm{E}-16$ & $6.57 \mathrm{E}-09$ & 0.784 & 0.071 \\
4 & $2.82 \mathrm{E}-08$ & 0.999 & 0.613 & 0.716 \\
5 & $2.76 \mathrm{E}-04$ & 0.999 & 0.511 & 0.999 \\
6 & $2.57 \mathrm{E}-05$ & 0.992 & 0.057 & 0.999 \\
7 & $8.73 \mathrm{E}-05$ & 0.89 & 0.017 & 0.999 \\
\hline
\end{tabular}

Sigmoidal membership functions with formula $\operatorname{sigm}(x,[\alpha, \gamma])=\frac{1}{1+e^{-\alpha(x-\gamma)}}$, were used to obtain the truth values in Table 3 , where $\alpha$ and $\gamma$ are real-valued parameters.

Suppose that experts determine that the parameters for $\mathrm{A}_{1}$ (Inflation) are $\alpha=-0.7659$ and $\gamma=5$; for $\mathrm{A}_{2}$ (GDP) are $\alpha=2.2976$ and $\gamma=2$; for $\mathrm{A}_{3}$ (Tax of exchange Peso/Dollar) are $\alpha=-0.9190$ and $\gamma=7$; for $\mathrm{A}_{4}$ (Demand) are $\alpha=6.5645 \mathrm{E}-4$ and $\gamma=32000$.

Let us compare the results of the predicate $p$, which is " $A_{1}$ and $A_{2}$ and $A_{3}$ imply $A_{4}$ " for the first and the second years, by using the probabilistic logic and the GMBCL. See Table 4.

The conjunction of $A_{1}, A_{2}$ and $A_{3}$ is the product of the truth values, according to Probabilistic logic system in Table 1, therefore, for the first year the truth value of the conjunction is $(0.01)(0.5)(0.987)=0.004935$. The truth value of $\mathrm{A}_{4}$ (Demand) for the first year is 0.557 , see Table 3. Now, we have to calculate the truth value of $i(0.004935,0.557)$, with the implication operator corresponding to the probabilistic logic system, that is the Reichenbach implication, see Table 1. $i(0.004935,0.557)=1-0.004935+(0.004935)(0.557)=$ 0.9978 .

The result for the second year is calculated similarly and is equal to 0.9078 . See table 4 .

On the other hand, with the GMBCL we applied the Geometric Mean, according to Table 1. The conjunction of the attributes $A_{1}, A_{2}$ and $A_{3}$ for the first year is $\sqrt[3]{(0.01)(0.5)(0.987)}=0.1703$. The truth value of the Natural implication with the GMBCL and formula appeared in Table 1 , is 0.7258 .

Similarly, for the second year the truth value of $\mathrm{p}$ is 0.4293

Table 4. Comparison of the truth value of the predicate $\mathrm{p}$ for the two first years in Table 3 and with two compatible logic systems: Probabilistic and GMBCL.

\begin{tabular}{lll}
\hline Logic system & $\begin{array}{l}\text { Truth value for the first } \\
\text { year }\end{array}$ & $\begin{array}{l}\text { Truth value for the } \\
\text { second year }\end{array}$ \\
\hline Probabilistic & 0.9978 & 0.9078 \\
GMBCL & 0.7258 & 0.4293 \\
\hline
\end{tabular}

Certainly, the calculus illustrated in Table 4 fulfills the proposition 3, where the truth value for the first year is bigger than the truth value for the second year in both compatible logic systems. The definitions of the operators used for calculation in Table 4 appear in Table 1 .

Therefore, the example 2 illustrates that from the point of view of the order, always it is maintaining when the calculus is made with compatible fuzzy logic systems.

Now, a new approach to the universal and the existential quantifier in an Archimedean-Compensatory Logic is introduced below.

\section{Definition 5}

Let be $X=\left\{x_{1}, \cdots, x_{n}\right\} \subset[0,1]$, the universal quantifier for the finite set $X$ and the continuous Archimedean tnorm $\mathrm{T}$, is defined by: $\forall_{\mathrm{T}} \mathrm{x}_{\mathrm{i}} \in \mathrm{X}=\mathrm{c}_{\mathrm{T}}\left(\mathrm{x}_{1}, \cdots, \mathrm{x}_{\mathrm{n}}\right)$. Let us call it Universal quantifier of no refutation.

\section{Definition 6}

Let be $X=\left\{x_{1}, \cdots, x_{n}\right\} \subset[0,1]$, the universal quantifier for the finite set $X$ and the conjunction operator of a CFL system based on quasi-arithmetic equations is defined by: $\forall_{\mathrm{C}} \mathrm{x}_{\mathrm{i}} \in \mathrm{X}=\mathrm{c}_{\mathrm{c}}\left(\mathrm{x}_{1}, \cdots, \mathrm{x}_{\mathrm{n}}\right)$. Let us call it Universal quantifier of affirmation.

\section{Definition 7}

Let be $X=\left\{x_{1}, \cdots, x_{n}\right\} \subset[0,1]$, the existential quantifier for the finite set $X$ and the continuous Archimedean tnorm $T$, is defined by: $\exists_{\mathrm{T}} \mathrm{x}_{\mathrm{i}} \in \mathrm{X}=\mathrm{d}_{\mathrm{T}}\left(\mathrm{x}_{1}, \cdots, \mathrm{x}_{\mathrm{n}}\right)$. Let us call it Existential quantifier of no refutation. 


\section{Definition 8}

Let be $\mathrm{X}=\left\{\mathrm{x}_{1}, \cdots, \mathrm{x}_{\mathrm{n}}\right\} \subset[0,1]$, the existential quantifier for the finite set $X$ and the CFL, is defined by: $\exists_{c} x_{i} \in$ $\mathrm{X}=\mathrm{d}_{\mathrm{c}}\left(\mathrm{x}_{1}, \cdots, \mathrm{x}_{\mathrm{n}}\right)$. Let us call it Existential quantifier of affirmation.

Let us note that the formulas for both universal quantifier operators, $\forall_{\mathrm{T}}$ and $\forall_{\mathrm{C}}$, are basically the same if they are generated by the same function, except for the index $\mathrm{n}=\operatorname{card}(\mathrm{X})$, which is a natural number. Bigger is $\mathrm{n}$, bigger is the evidence for the person about the falseness of the truth value of the universal predicate, and it is also the linguistic modifier necessary for compensation. Hence, this relationship is the basis of the unification of the t-norm theory and the CFL theory. The effect of applying the $\forall_{\mathrm{T}}$ operator is the conversion of the fuzzy predicates in crisp predicates, because if $n$ increases and $\forall_{\mathrm{T}} \mathrm{x}_{\mathrm{i}} \in \mathrm{X}$ isn't absolutely true, then, this predicate becomes falser.

Besides, if the simple predicates are all true, then $\forall_{\mathrm{T}} \mathrm{X}_{\mathrm{i}} \in \mathrm{X}$ will be always true and the logic system tends to be a bivalent logic system.

On the other hand, the effect to apply $\forall_{C}$ is to maintain the regularity of the truth values of the simple predicates, due to the idempotency property. The tendency to this operator is to affirm the proposition.

This pair of both tendencies of behavior of the persons describes better the preferences instead of the use of one of them exclusively.

Therefore, the name of the no refutation operator is because it has the tendency to reject the acceptance that the propositions are true as well as the affirmation operator tends to confirm the truth value of every proposition.

On the other hand, CFL is a less drastic approach, where the calculus over the expressions tends to affirm, instead to refute. The property which justifies this statement is the set of inequalities:

$\forall_{\mathrm{T}} \mathrm{x} \leq \min \{\mathrm{x}\} \leq \forall_{\mathrm{C}} \mathrm{x} \leq \exists_{\mathrm{C}} \mathrm{x} \leq \max \{\mathrm{x}\} \leq \exists_{\mathrm{T}} \mathrm{x}$

Let us note that inequalities (11) include the max and min of the truth value vector, which correspond to the Zadeh max-min logic system.

\section{Remark 4}

In the set of inequalities 11 , the inequality $\forall_{C} \mathrm{x} \leq$ $\exists_{C} \times$ can be easily proved for the case of Eqs. (7) and (8) defining the conjunction and the disjunction.

It is well known that the mean represented in Eq. (7) for $p \leq 0$ is less or equal to the arithmetic mean (for $p=1$ ), hence.

$$
\begin{aligned}
\left(\frac{\sum_{i=1}^{n} x_{i}^{p}}{n}\right)^{1 / p}+ & \left(\frac{\sum_{i=1}^{n}\left(1-x_{i}\right)^{p}}{n}\right)^{1 / p} \\
& \leq \frac{\sum_{i=1}^{n} x_{i}}{n}+\frac{\sum_{i=1}^{n}\left(1-x_{i}\right)}{n}=1
\end{aligned}
$$

Therefore, $\left(\frac{\sum_{i=1}^{n} x_{i}^{p}}{n}\right)^{1 / p} \leq 1-\left(\frac{\sum_{i=1}^{n}\left(1-x_{i}\right)^{p}}{n}\right)^{1 / p}$

and hence, $\forall_{\mathrm{C}} \mathrm{x} \leq \exists_{\mathrm{C}} \mathrm{x}$.

Hence, an Archimedean-Compensatory Logic has a property of affirmation and refutation at the same time, which are complementary properties.

Below, we summarize the two main properties demonstrated above in this paper as a consequence of propositions 1, 2 and 3:

\section{Properties}

1. If $\forall_{\mathrm{T}} \mathrm{xp} \mathrm{p}_{1 \mathrm{~T}}(\mathrm{x})<\forall_{\mathrm{T}} \mathrm{x} \mathrm{p}_{2 \mathrm{~T}}(\mathrm{x})$ then $\forall_{\mathrm{C}} \mathrm{x} \mathrm{p}_{1 \mathrm{C}}(\mathrm{x})<$ $\forall_{\mathrm{C}} \mathrm{x} \mathrm{p}_{2 \mathrm{C}}(\mathrm{x})$ and vice versa

2. If $\exists_{\mathrm{T}} \mathrm{x} \mathrm{p}_{1 \mathrm{~T}}(\mathrm{x})<\exists_{\mathrm{T}} \mathrm{x} \mathrm{p}_{2 \mathrm{~T}}(\mathrm{x})$ then $\exists_{\mathrm{C}} \mathrm{x} \mathrm{p}_{1 \mathrm{C}}(\mathrm{x})<$ $\exists_{C} x p_{2 C}(x)$ and vice versa

Where the quartet

$\left(\forall_{\mathrm{T}} \mathrm{xp}_{\mathrm{T}}(\mathrm{x}), \forall_{\mathrm{C}} \mathrm{xp}_{\mathrm{C}}(\mathrm{x}), \exists_{\mathrm{C}} \mathrm{xp}_{\mathrm{C}}(\mathrm{x}), \exists_{\mathrm{T}} \mathrm{xp}_{\mathrm{T}}(\mathrm{x})\right)$ is formed by the following four elements below:

1. $\forall_{\mathrm{T}} \mathbf{x} \boldsymbol{p}_{\boldsymbol{T}}(\boldsymbol{x})$ is called the no refutation value of the necessity of $p(x)$.

2. $\forall_{\mathrm{C}} \mathbf{x} \boldsymbol{p}_{\boldsymbol{C}}(\boldsymbol{x})$ is called the affirmation value of the necessity of $p(x)$.

3. $\exists_{C} \mathbf{x} \boldsymbol{p}_{\boldsymbol{C}}(\boldsymbol{x})$ is called the affirmation value of the possibility of $p(x)$.

4. $\exists_{\mathrm{T}} \mathbf{x} \boldsymbol{p}_{T}(\boldsymbol{x})$ is called the no refutation value of the possibility of $p(x)$.

These four operators defined above, are other approaches to the well known concepts of Necessity and Possibility appeared in Modal Logic and included in the possibility fuzzy logic theory, see Refs. 9-10.

\section{Example 3}

Let us calculate the four quantifiers in the ArchimedeanCompensatory logic based on probabilistic logic system and GMBCL for the truth values appeared in Table 3.

This is an example to illustrate the use of this new theory for Knowledge Discovery.

Let us consider the question: Is a high demand equivalent to a high GDP more significant than a low inflation equivalent to a high demand?, for answer this question we will consider the results in Table 3 and the predicate 'high GDP $\leftrightarrow$ high Demand' or symbolically $\mathrm{A}_{2} \leftrightarrow \mathrm{A}_{4}$ and 'low inflation $\leftrightarrow$ high Demand' or symbolically $\mathrm{A}_{1} \leftrightarrow \mathrm{A}_{4}$.

Now we will consider the universal quantifiers for every object of the predicate evaluated in Table 2, with the truth values appeared in Table $3, \mathrm{p}_{1}=$ 'for every objects 
of attribute $A_{2}$ is equivalent to $A_{4}$ ' $p_{2}=$ 'for every objects of attribute $A_{1}$ is equivalent to $A_{4}$ '.

The way for calculating the affirmation value of the possibility of proposition $\mathrm{A}_{2} \leftrightarrow \mathrm{A}_{4}$, for instance, is detailed below:

1. From the truth values in Table 3, calculate the truth value of the equivalence operator of GMBCL, by using the formula of compensatory operator defined in Table 1.

That is to say, apply formula $e\left(\mathrm{x}_{1}, \mathrm{x}_{2}\right)=$ $\sqrt{i\left(\mathrm{x}_{1}, \mathrm{x}_{2}\right) \cdot i\left(\mathrm{x}_{2}, \mathrm{x}_{1}\right)}$, where $i\left(\mathrm{x}_{1}, \mathrm{x}_{2}\right)$ is the Natural implication of the GMBCL, $x_{1}$ is every truth value of $A_{2}$ in Table 3 and $x_{2}$ is every truth value of $A_{4}$ in Table 3 .

They are: $e(0.5,0.557)=0.5, e(0.99,0.388)=$ $0.4559, \quad e(6.57 \mathrm{E}-09,0.071)=0.8564$, $e(0.999,0.716)=0.6744, e(0.999,0.999)=0.9684$, $e(0.992,0.999)=0.9391, e(0.89,0.999)=0.8053$.

The result is the set of truth values after we applied the formula of compensatory equivalence between $\mathrm{A}_{2}$ and $\mathrm{A}_{4}$ for every year in the Mexican Economy.

2. Now, apply to this set of truth values the formula of Universal quantifier of affirmation, according to Definition 6. That is to say, calculate $c(0.5,0.4559,0.8564,0.6744,0.9684,0.9391,0.8053)$ which is the Geometric Mean, see (9), of these values and equal to 0.7159 .

Similarly, we can obtain the truth value of $\mathrm{p}_{2}$, for the GMBCL and the predicate $p$ equal to $A_{1} \leftrightarrow A_{4}$.

Later, repeat the operations explained above, for the two predicates and the Probabilistic Logic system.

The truth value of the affirmation value of the necessity for $\mathrm{p}_{1}(\mathrm{x})$ is 0.7159 by using the GMBCL and for $\mathrm{p}_{2}(\mathrm{x})$ is 0.0853, therefore, is much more significant the correlation between demand and GDP than the correlation between demand and inflation.

On the hand, the same calculus with the no refutation value of the necessity of $p_{1}(x)$ and $p_{2}(x)$, are 0.0105 and $1.0779 \mathrm{e}-015$, respectively, therefore the same order is maintained by using the probabilistic logic.

Repeat the same for the Existential quantifier.

In both cases, with GMBCL and the probabilistic logic, they are basically the same, except for the purpose of the calculus, where the no refutation value of the necessity is used to the purpose of no refute and the affirmation value of the necessity is used to affirm the necessity of propositions.

Similarly the existential quantifiers are used to affirm or to no refute the possibility of propositions.

Therefore, for this problem it is better to associate high GDP to high demand than to associate it to low inflation.

Summarizing, we can conclude that the quartet of formulas of necessity and possibility allow to make a better decision than by using just one of them, because the four truth values express four different point of view of the problem.

\section{Concluding Remarks}

This paper introduces novelty approaches to the fuzzy logic theory. First, the concept of fuzzy logic system prevailed over the use of isolated operators. From this point of view the continuous Archimedean t-norm and tconorm are defined like a logic system, and the concept of Archimedean logic is expressed for the first time.

Here we introduced the concept of compatibility between two fuzzy systems based on the unchanged order of preferences between two vectors evaluated in a predicate.

Also we showed that a continuous Archimedean logic generated by a monotone real valued function has an equivalent CFL system generated by the same function. On the other hand, we proved that two truth value vectors maintain the same order when they are evaluated in certain predicate, for one continuous Archimedean logic generated by a function and its corresponding CFL generated by the same function. Therefore, every Archimedean logic system has a compatible CFL system.

Based on the ideas indicated above, we defined a new logic system, named Archimedean-Compensatory logic, which includes a quartet of universal and existential operators of an Archimedean logic and its compatible CFL. The universal quantifier is defined like a conjunction of the elements and the existential operator is defined like the disjunction.

The quartet formed by one Universal quantifier of no refutation, one Universal quantifier of affirmation, one Existential quantifier of no refutation and one Existential quantifier of affirmation, indicates two semantic approaches, they are the approach no refutation of the pair formed by the Universal quantifier of no refutation and the Existential quantifier of no refutation and the approach affirmation of the pair formed by the Universal quantifier of affirmation and the Existential quantifier of affirmation.

Also, for some logic proposition we defined a quartet formed by that we called the no refutation value of the necessity of the proposition, the affirmation value of the necessity of the proposition, the no refutation value of the possibility of the proposition and the affirmation value of the possibility of the proposition.

Some examples of this theory and its application to real world problems illustrated the definitions and propositions used in this paper. 


\section{References}

1. M. Detyniecki, Mathematical aggregation operators and their application to video querying, $\mathrm{PhD}$ thesis, Berkeley University, Berkeley, United States, 2003.

2. R. A. Espín, E. Fernández and E. González, Compensatory Fuzzy Logic: A Frame for Reasoning and Modeling Preference Knowledge in Intelligent Systems, in Soft Computing for Business Intelligence, (Springer Berlin Heidelberg, 2014), pp. 3-23.

3. I. B. Turksen, Interval-valued fuzzy sets and Compensatory AND', Fuzzy Sets and Systems. 51(3) (1992) 295-307.

4. I. B. Turksen, Compensatory "AND" in An ontological and epistemological perspective of fuzzy set theory, (Elsevier, 2005), pp. 267-288.

5. E. P. Klement, R. Mesiar, and E. Pap, On the relationship of associative compensator y-operators to triangular norms and conorms. International Journal of Uncertainty, Fuzziness and Knowledge-based Systems 4(2)(1996) 129-144.

6. R. Mesiar, Compensatory operators based on triangular norms and conorms. in: Proc. EUFIT Conference, (Elite Foundation, Aachen 1995), pp. 131-135.
7. A. Kolesárová and M. Komorníková, Triangular normbased iterative compensatory operators, Fuzzy Sets and Systems, 104(1)(1999) 109-120.

8. D. Gómez, and J. Montero, A discussion on aggregation operators, Kybernetika 40 (1) (2004)107-120.

9. D. Dubois and H. Prade. Possibility theory, probability theory and multiple-valued logics: A clarification. Annals of mathematics and Artificial Intelligence 32(1-4) (2001) 35-66.

10. D. Dubois, F. Esteva, L. Godo and H. Prade, Fuzzy-Set Based Logics. A history oriented presentation of their main development, in The Many Valued and Nonmonotonic Turn Logic, Handbook of the History of Logic (Elsevier, 2007), pp. 325-449.

11. A. Rosete, T. Ceruto, R. A. Espin and J. Marx-Gomez, A General Method for Knowledge Discovery Approach using Compensatory Logic and metaheuristics, in Towards a Trans-disciplinarian Technology for Business Intelligence: Gathering Knowledge Discovery, Knowledge Management and Decision Making, (Shaker Verlag, 2011), pp. 240-270. 